\title{
Mathematical and numerical modeling of the effect of input-parameters on the flushing efficiency of plasma channel in EDM process
}

\author{
Mohammadreza Shabgard ${ }^{\mathrm{a}, *}$, Reza Ahmadi ${ }^{\mathrm{a}}$, Mirsadegh Seyedzavvar ${ }^{\mathrm{a}}$, Samad Nadimi Bavil Oliaei ${ }^{\mathrm{b}}$ \\ a Department of Mechanical Engineering, Tabriz University, 29 Bahman Boulevard, Tabriz 51666-14766, Iran \\ ${ }^{\mathrm{b}}$ Department of Mechanical Engineering, Faculty of Engineering, Bilkent University, Bilkent, Ankara 06800, Turkey
}

\section{A R T I C L E I N F O}

\section{Article history:}

Received 14 February 2012

Received in revised form

15 October 2012

Accepted 16 October 2012

Available online 1 November 2012

Keywords:

Electrical discharge machining

Numerical analysis

Mathematical modeling

Plasma flushing efficiency

Recast layer thickness

\begin{abstract}
A B S T R A C T
In the present study, the temperature distribution on the surface of workpiece and tool during a single discharge in the electrical discharge machining process has been simulated using ABAQUS code finite element software. The temperature dependency of material properties and the expanding of plasma channel radius with time have been employed in the simulation stage. The profile of temperature distribution has been utilized to calculate the dimensions of discharge crater. Based on the results of FEM and the experimental observations, a numerical analysis has been developed assessing the contribution of input-parameters on the efficiency of plasma channel in removing the molten material from molten puddles on the surfaces of workpiece and tool at the end of each discharge. The results show that the increase in the pulse current and pulse on-time have converse effects on the plasma flushing efficiency, as it increases by the prior one and decreases by the latter one. Later, the introduced formulas for plasma flushing efficiency based on regression model were utilized to predict the cardinal parameter of recast layer thickness on the electrodes which demands expensive empirical tests to be obtained.
\end{abstract}

(c) 2012 Elsevier Ltd. All rights reserved.

\section{Introduction}

Electrical discharge machining (EDM) is a competent machining process used in the machining of hard materials and manufacturing of workpieces with a complicate profile $[1,2]$. Nevertheless, the low level of material removal rate, tool wear, and surface deficiencies of the machined workpiece are considered to be the major obstacles of this process being highly dependent on a factor called plasma flushing efficiency (\%PFE). Broadly speaking, plasma flushing efficiency could be considered as the key element affecting the outcomes of EDM process, such as material removal rate, tool wear ratio, and surface texture features [3,4]. Plasma flushing efficiency itself is dependent on the input parameters of the process among which pulse current and pulse on-time are the most prominent ones [5].

Considering the fact that the quality of an ED machined surface has been becoming more and more important to satisfy the increasing demands of sophisticated component performance, longevity and reliability; several studies have been carried out to determine appropriate ED machining parameter combinations with respect to the surface integrity. However, these studies were based on the use of experimental approaches and statistical

\footnotetext{
* Corresponding author. Tel./fax: +984113315697.

E-mail address: mrshabgard@tabrizu.ac.ir (M. Shabgard).
}

analyses. In few studies, pure theoretical approaches have been proposed to estimate the outputs of EDM process, using FE or analytical methods.

Ben Salah et al. [6] presented a numerical model to study the temperature distribution at EDM process and used the thermal results for predicting the material removal rate and the total surface roughness. In their study, the fraction of the generated heat entering the workpiece was considered equal to 0.08 . They reported that taking into account the temperature dependence of the conductivity is of crucial importance to the accuracy of the numerical results and gives the better correlation with experimental observations. Marafona and Chousal [7] employed a FEA model to estimate the surface roughness and the removed material from both anode and cathode. They reported that the anode material removal efficiency is smaller than that of the cathode because there is a high amount of energy going to the anode and also a fast cooling of this material. They explained that this phenomenon can be explained by the differences of thermal conductivity of the cathode and anode. Kansal et al. [8] developed a model to calculate the temperature distribution in the workpiece material by employing the ANSYS software in the powder mixed electrical discharge machining. In their presented model it has been assumed that $9 \%$ of the total heat was absorbed by the workpiece. They utilized the results of finite element simulation to estimate the material removal rate of workpiece. The usage of introduced temperature dependent material properties was one 


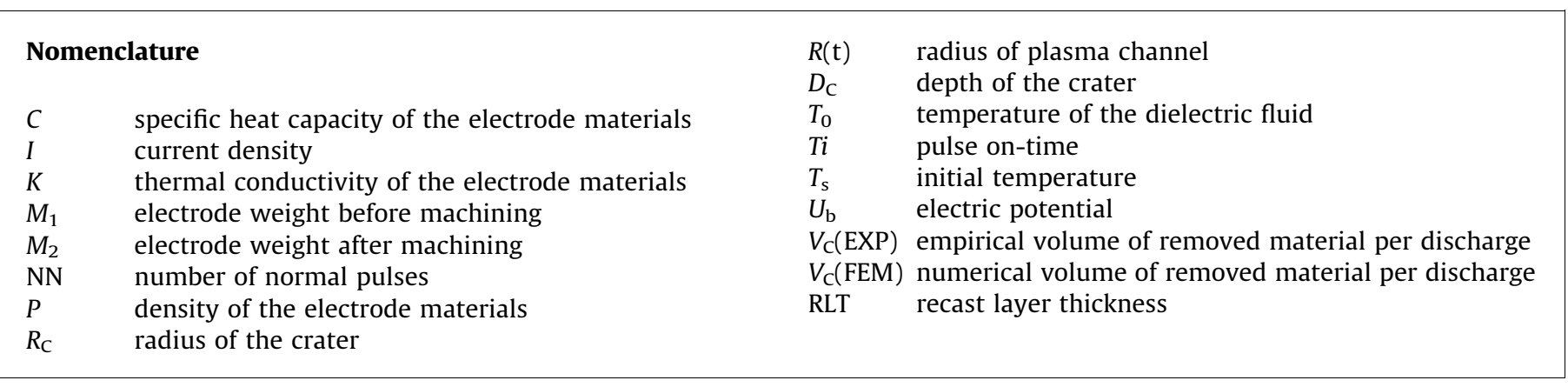

of the major features of their model which led to a better accuracy in predicting of MRR parameter. Joshi and Pande [9] introduced an intelligent process modeling and optimization of EDM process.

One of the major impediments on the way of proposing a unique method for predicting the output parameters of EDM process is the recondite behavior of plasma channel and its efficiency in removing molten material from the molten puddle. For instance Dibitoto et al. [5] reported that the fraction of heat going to the cathode and anode were $18.3 \%$ and $8 \%$, respectively, with remainder lost to the dielectric. In other study, Hashimoto et al. [10] reported a distribution of $34 \%$ and $48 \%$ discharge energy into the cathode and anode, respectively. Having modeled of heat distribution in anode and cathode in micro-EDM, Yeo et al. [11] proposed a fraction of $14 \%$ and $39 \%$ of discharge energy to the anode and cathode, respectively.

In the present study, the different proposed values for fraction of heat dispersed into the workpiece $\left(f_{\mathrm{c}}\right)$ and tool $\left(f_{\mathrm{a}}\right)$ have been conducted and the best values for $f_{\mathrm{c}}$ and $f_{\mathrm{a}}$, which resulted in the better proximity of numerical results to the experimental observations, were calculated based on the regression modeling of the outputs of finite element modeling of heat distribution during the discharge and the experimental observations of material removal rate and surface texture feature of recast layer thickness (RLT). Later, the influence of pulse on-time (Ti) and pulse current (I) were detailed based on numerical results and experimental observations.

\section{Experimental procedure}

The workpiece and tool materials used in this study were AISI H13 tool steel and forged commercial pure copper, respectively. The main mechanical and physical properties of such materials at different temperatures are given in Table 1.

The experiments were performed on a die sinking EDM machine (CHARMILLES ROBO-FORM200) which operates with an iso-pulse generator. During the EDM experiments, the electrode and the workpiece were submerged in a bath of Oil Flux ELF2 which acted as the dielectric fluid. Machining tests were carried out at eight pulse current settings as well as five pulse on-time settings. Each machining test was performed for $15 \mathrm{~min}$. The machining settings and the detail of experimental procedure are listed in Table 2.

For on-line registering the number of different kind of pulses (normal pulses, arc discharges and open circuits) during the EDM process, an oscilloscope (Hitachi VC-6524) of storage type and an electronic circuit were employed to capture the gap voltage and current variations against time, which were then transferred and stored on a PC hard disk through a serial cable and port connection. Additionally, in order to count the number of each type of pulses, a program in FORTRAN language was written and linked to the pulse monitoring software (ITM).

A digital balance (CP2245-Surtorius) with a resolution of $0.1 \mathrm{mg}$ was used for weighing the workpieces before and after the machining process.

The recast layer is so infiltrated with carbon that has a separate, distinct structure, totally distinguishable from the parent material in the scanning electron microscopy (SEM) images [14]. Consequently, the amount of recast layer thickness has been measured by measuring this layer's thickness at 30 different points by SEM and accounting for their average. So the machined specimens were sectioned transversely by a wire electrical discharge machine and prepared under a standard procedure for metallographic observation. Etching was performed by immersing the specimens in $5 \%$ Nital reagent. VEGA $\backslash$ TESCAN scanning electron microscopy was employed at this stage.

Table 2

Experimental test conditions.

\begin{tabular}{ll}
\hline Generator type & Iso-pulse (ROBOFORM 200) \\
Dielectric fluid & Oil Flux ELF2 \\
Flushing type & Normal submerged \\
Power supply voltage $(\mathrm{V})$ & 200 \\
Reference voltage $(\mathrm{V})$ & 70 \\
Pulse current $(\mathrm{A})$ & $8,12,16,20,24,32,48$ \\
Polarity & Positive \\
Pulse on-time $(\mu \mathrm{s})$ & $12.8,25,50,100,200$ \\
Pulse interval $(\mu \mathrm{s})$ & 6.4 \\
Tool material & Commercial pure copper \\
Tool shape & Cylindrical $(\emptyset 18.3 \mathrm{~mm}$ and $L=20 \mathrm{~mm})$ \\
\hline
\end{tabular}

Table 1

Mechanical and physical properties of electrodes [12,13].

\begin{tabular}{|c|c|c|}
\hline Property & AISI H13 workpiece & Cupper tool \\
\hline Density $\left(\mathrm{kg} / \mathrm{dm}^{3}\right)$ & $3007 \times \mathrm{T}-385.16 \times \mathrm{T} 2$ & $8.923+0.000008 \times \mathrm{T}-0.000001 \times \mathrm{T} 2$ \\
\hline Specific heat $(\mathrm{J} /(\mathrm{kg} \mathrm{K}))$ & $7947.4+27.346 \times \mathrm{T}-0.0218 \times \mathrm{T}^{\wedge} 2$ & $381.5+0.1266 \times \mathrm{T}-0.000035 \mathrm{~T}^{\wedge} 2$ \\
\hline Modulus of elasticity $\left(\mathrm{N} / \mathrm{mm}^{2}\right)$ & $216.13-0.0555 \times \mathrm{T}-5 \mathrm{E}-05 \times \mathrm{T}^{\wedge} 2$ & - \\
\hline Electrical resistivity $\left(\mathrm{Ohm} \mathrm{mm}^{2} / \mathrm{m}\right)$ & $0.5109+0.0004 \times \mathrm{T}+5 \mathrm{E}-07 \mathrm{~T}^{\wedge} 2$ & - \\
\hline Thermal conductivity $(\mathrm{W} / \mathrm{m} \mathrm{K})$ & $21.314+0.0354 \mathrm{~T}-6 \mathrm{E}-05 \mathrm{~T}^{\wedge} 2+3 \mathrm{E}-08 \mathrm{~T}^{\wedge} 3$ & $401.4-0.06248 \times \mathrm{T} ; \mathrm{T}<1084{ }^{\circ} \mathrm{C} 86.75+0.09267 \times \mathrm{T}-0.000024 \times \mathrm{T} 2 ; \mathrm{T}>1084{ }^{\circ} \mathrm{C}$ \\
\hline Liquidus temperature $\left({ }^{\circ} \mathrm{C}\right)$ & 1454 & 1084.88 \\
\hline Latent heat of fusion $(\mathrm{J} / \mathrm{kg})$ & $2.58 \mathrm{E} 05$ & 205 \\
\hline
\end{tabular}




\section{FE simulation and regression modeling}

\subsection{Mathematical fundaments}

Generally, the differential equation for heat transfer without internal heat generation term is used as the base of the thermal modeling for describing the state of heat distribution during each discharge [15].

$$
(1 / r)(\partial / \partial r)(r(\partial T / \partial r))+\partial^{2} T / \partial z^{2}=(\rho \cdot C / k)(\partial T / \partial t)
$$

where $T$ is the temperature, $t$ is the time, $\rho, k$ and $C$ are respectively the density, thermal conductivity and specific heat capacity of the electrode materials. Finally, $r$ and $z$ are the coordinate axes as illustrated in Fig. 1.

The considered domain is a small cylindrical portion of the electrodes around the spark. In the domain the heat flux is applied on the surface $B_{1}$ (Fig. 1 ).when $t>0$

$B C_{S}: k(\partial T / \partial z)=\left\{\begin{array}{l}h_{\mathrm{c}}\left(T-T_{0}\right) r>R \\ q(r) r \leq R \text { on } B_{1} \\ 0 \text { for off-time }\end{array}\right.$

and

$(\partial T / \partial n)=0$ on $B_{2}, B_{3}, B_{4}$

here, $q(r)$ is the quantity of heat flux entering into the electrodes; $R$ is the radius of spark (plasma channel).

The initial temperature $T_{\mathrm{s}}$ can be taken the temperature $T_{0}$ of the dielectric fluid in which the electrodes are submerged. Thus,

$T_{S}=T_{0} \quad$ at $\quad t=0$

In addition, the following assumptions have been considered:

1. The model is developed for a single spark.

2. The electrodes are considered as semi-infinite body since the volume of removed material is much smaller than the volume of the electrodes.

3. Since the precise position of each spark is random, the effect of single discharge is useful in developing an inside into the temperature distribution at electrodes during each discharge. Consequently, the thermal effects of successive sparks are neglected and the crater is individually identified to simplify the simulation process $[7,16]$.

4. The magnitude of the heat flux incident on the electrodes is independent of the affected surface profile.

5. The effects of spark gap on discharge characteristics are negligible.

6. The phase changes during the analysis are neglected.

7. The craters formed on the electrodes due to each discharge are assumed to have circular paraboloid geometries.

8. The redeposit of recast layer in the crater after each spark is considered uniform.

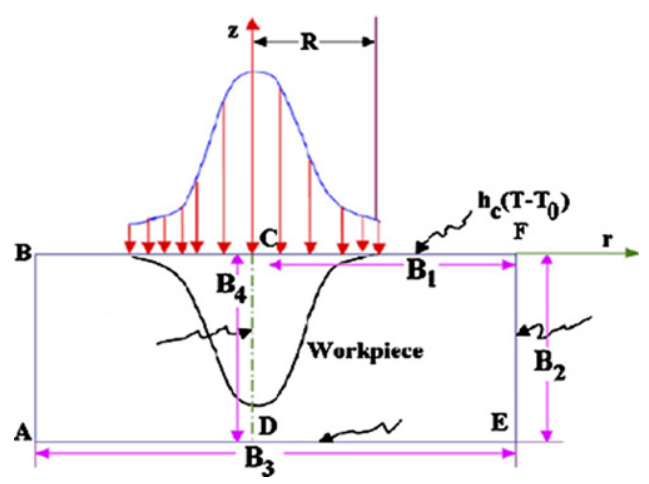

Fig. 1. Thermal model of the EDM process.
In this research, it was decided to determine the radius of the discharge channel (spark) based on the work from Ikai and Hashiguchi [17]. In particular, this radius is called the equivalent heat input radius and it can be expressed as a function of the pulse current $(I)$ and the pulse on-time (Ti) as shown in the following equation:

$R(t)=2.04 \times I^{0.43} \times T i^{0.44}$

where $R(t)$ is the radius of plasma channel $(\mu \mathrm{m}), I$ is pulse current $(I)$ and $T i$ is pulse on-time ( $\mu$ s).

Gaussian heat distribution has been employed in the present work as it provides more accurate results than the uniform disc heat source [15]. The following equation represents the heat flux $q(r)$ at radius $r[18]$ :

$q(r)=4.5\left(F U_{b} I / \pi R^{2}(t)\right) \times \exp -4.5(r / R(t))^{2}$

where $U_{\mathrm{b}}$ is the breakdown (discharge) voltage, $I$ is the pulse current $(A)$ and $f$ is the energy distribution to the electrodes.

The selected element type in the FE analyses is a 10-node quadratic heat transfer tetrahedron. A subroutine has been written for applying the heat flux developing with time on the work domains. The temperature profile obtained from the FEM analysis is used to calculate the amount of material removed from the specimen exposed to the heat flux [19].

\subsection{Application of FEM in thermal modeling of EDM}

The implemented numerical analysis carried out in this study was based on the Gaussian distributed heat flux during each spark of the EDM process. The standard finite element software ABAQUS/CAE was employed for simulating the temperature distribution in the electrodes during the discharge process. One of the special features of this software is its ability in considering the temperature dependent material properties for specimen. Consequently, the temperature dependency of material properties was taken into account in the simulation stage. This consideration increases the compatibility of predicted values with the experimental observation. The latent heat of melting, which was considered one of the major features of electrodes and had a crucial effect on the result of simulation, was considered in the numerical analysis.

Based on ABAQUS code, a transient thermal analysis was conducted to calculate the temperature field in the electrodes and to study the heat distribution with respect to different machining parameter settings. The size of domain for the thermal analyses was dependent on the input parameters of EDM process since the radius of discharge channel was determined based on the machining settings (Eq. (5)). The numerical analyses were performed using a 10-node quadratic heat transfer tetrahedron (DC3D10) mesh with the global size of $13 \mu \mathrm{m}$. Approximately 45,000 elements have been generated on the instance.

For applying the heat flux varying with time on the work domain, a discontinuous method for discretization in time has been utilized by employing an arbitrary polynomial order. Let $t_{0}<t_{1}<t_{2}<\ldots<t_{\mathrm{n}}=T_{\mathrm{on}}$ be a sequence of times and a partition of time interval $\left[0, T_{\text {on }}\right]$. A 'dflux' subroutine was used to calculate the heat flux exposed on the discharge location of instance. The temperature profiles obtained from the FEM analysis were used to calculate the amount of material removed from the specimen.

\subsection{The morphology of crater cavity}

To calculate the cavity volume at the end of each discharge, the isothermal counter at the melting temperature was drawn through the $\left(x_{\mathrm{i}}, z_{\mathrm{i}}\right)$ points obtained by the FEM. Fig. 2 represents 
this counter as well the parabolic crater cavity form suggested by Ikai and Hashigushi [17].

Using the $\left(x_{\mathrm{i}}, z_{\mathrm{i}}\right)$ nodes resulted from the FEM and employing the equation (Eq. (7)) introduced by Joshi and Pande [9] the cavity volume is calculated.

$V_{C}(\mathrm{FEM})=\pi\left(\left(x_{i}+x_{i+1}\right) / 2\right)^{2}\left(z_{i+1}-z_{i}\right)$

where $V_{C}($ FEM $)$ is the volume of the crater and $x_{\mathrm{i}}$ and $z_{\mathrm{i}}$ the coordinates of the node of the isothermal counter.

It has been proved that the crater volume obtained by using the Eq. (7) is equal to the volume by the assumption of a circular parabolic geometry for the crater [19] since the parabolic counter fits the isothermal counter passing through the $\left(x_{\mathrm{i}}, z_{\mathrm{i}}\right)$ coordinates. This geometry is shown in Fig. 2.

The theoretical crater volume defined by the parabolic geometry is described by the following equation:

$V_{C}(\mathrm{FEM})=\frac{1}{2} \pi D_{C} R_{C}^{2}$

where $D_{C}$ and $R_{C}$ are the depth and radius of the crater, respectively.

\subsection{Regression modeling}

In a unique and innovative approach towards the calculation of the fraction of heat distributed to the cathode, anode, and dielectric, a combination of the effects of dispersed energy into

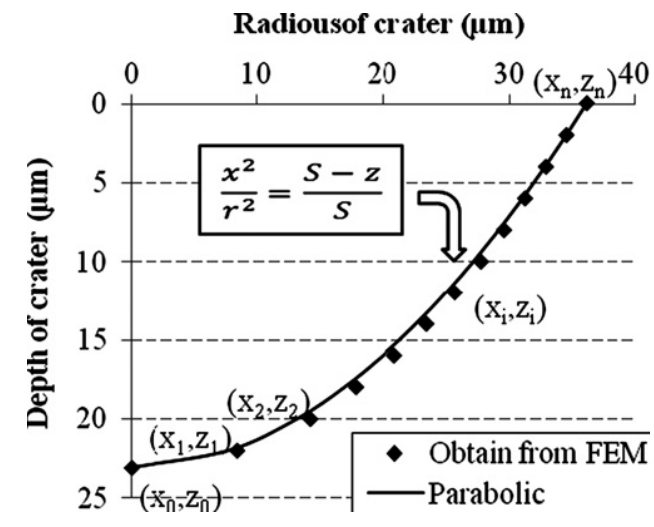

Fig. 2. Iso-thermal counter at the melting temperature. the electrodes and the amount of molten material ejected from the molten puddle at the end of discharge have been taken into account. Having this in mind, the value of the energy distributions to the cathode $\left(f_{\mathrm{c}}\right)$ and anode $\left(f_{\mathrm{a}}\right)$ have been calculated based on the results of FEM of thermal distribution into the electrodes and through considering the experimental values of recast layer thickness at a number of different pulse settings. At this stage, the trial and error method, an empirical method to test a hypothesis or procedure by repeating an experiment until the chance of error or outcome is known to some degree of reliability, have adapted to achieve the values of $f_{\mathrm{c}}$ and $f_{\mathrm{a}}$ at different pulse setting (Tables 3 and 4). Based on this approach, recast layer thickness of EDMed electrodes have been measured after preparation stages scanning and optical microscopes. An initial value have been selected for $f_{\mathrm{c}}$ and $f_{\mathrm{a}}$ for different settings, Eqs. (9-11) have been used to calculate the value of $\operatorname{RLT}_{\mathrm{FEM}}$. The calculated values have been compared with the experimental values of recast layer thickness ( $\mathrm{RLT}_{\mathrm{EXP}}$ ) and the value of $f_{\mathrm{c}}$ and $f_{\mathrm{a}}$ will be changed in a way that the RLT $\mathrm{FEM}_{\mathrm{Fe}}$ approximate the $\mathrm{RLT}_{\mathrm{EXP}}$ within the acceptable tolerance. Later, the regression modeling through the utilization of NLREG software handled in order to elicit the relationship between the energy distribution to the electrodes and the pulse current and pulse on-time, accounting for the fact that the amount of heat distributed into the cathode and anode has a close proximity with the pulse settings and differs with input parameters of EDM process. The following correlations represent the procedure of calculating the $f_{\mathrm{c}}$ and $f_{\mathrm{a}}$.

The plasma flushing efficiency (\%PFE), that was introduced in previous studies as the metal removal efficiency [7], is defined as the ratio of the actual volume of removed material per pulse versus the theoretical volume of melted material per pulse. Its value is described by the following equation:

$\% \mathrm{PFE}=100 \times V_{C}(\mathrm{EXP}) / V_{C}(\mathrm{FEM})$

The values of $V_{\mathrm{C}}(\mathrm{EXP})$ has been calculated by the following equation:

$V_{C}(\mathrm{EXP})=\left(M_{1}-M_{2}\right) /(N N \times \rho)$

where $M_{1}$ and $M_{2}$ are the weight of electrodes before and after machining, respectively, and $\mathrm{NN}$ is the number of normal pulses at the end of machining period (Table 5). This value has been achieved by utilization of an oscilloscope (Hitachi VC-6524) of storage type and an electronic circuit to capture the gap voltage

Table 3

Experimental data and theoretical features employed in regression modeling of $f_{\mathrm{c}}$.

\begin{tabular}{|c|c|c|c|c|c|c|c|c|c|}
\hline$I(\mathrm{~A})$ & $T i(\mu \mathrm{s})$ & $V_{\mathrm{c}}(\mathrm{EXP})\left(\mu \mathrm{m}^{3}\right)$ & $\operatorname{RLT}_{\text {EXP }}(\mu \mathrm{m})$ & $f_{\mathrm{c}}$ & $R_{\mathrm{C}}(\mu \mathrm{m})$ & $D_{\mathrm{C}}(\mu \mathrm{m})$ & $V_{\mathrm{c}}(\mathrm{FEM})\left(\mu \mathrm{m}^{3}\right)$ & $\operatorname{RLT}_{\mathrm{FEM}}(\mu \mathrm{m})$ & PFE\% \\
\hline \multirow[t]{4}{*}{8} & 12.8 & 2654.422 & 7.3 & 5.35 & 20.45 & 11.34 & 7449.37 & 7.30 & 35.627 \\
\hline & 25 & 5177.019 & 8.6 & 6.8 & 25.07 & 13.84 & $13,663.58$ & 8.597 & 37.884 \\
\hline & 50 & $10,224.18$ & 19.3 & 8.83 & 41.85 & 23.02 & $63,330.97$ & 19.303 & 16.147 \\
\hline & 100 & $18,146.54$ & 23.4 & 10.7 & 51.44 & 27.77 & $115,424.4$ & 23.403 & 15.724 \\
\hline \multirow[t]{4}{*}{16} & 12.8 & 6724.204 & 7.7 & 4.5 & 26.41 & 13.84 & $15,163.27$ & 7.701 & 44.359 \\
\hline & 25 & $14,651.34$ & 10.7 & 5.57 & 34.83 & 18.39 & $35,043.59$ & 10.701 & 41.808 \\
\hline & 50 & $30,980.84$ & 17.75 & 6.97 & 49.17 & 25.91 & $98,398.33$ & 17.751 & 31.489 \\
\hline & 100 & $65,908.09$ & 22.5 & 8.9 & 62.94 & 33.09 & $205,906.5$ & 22.498 & 32.011 \\
\hline \multirow[t]{4}{*}{24} & 12.8 & $10,894.53$ & 6.5 & 4.18 & 29.3 & 14.58 & 19661.32 & 6.500 & 55.418 \\
\hline & 25 & $24,588.52$ & 8.3 & 5.23 & 38.01 & 19.14 & 43436.77 & 8.301 & 56.629 \\
\hline & 50 & $52,398.1$ & 14.2 & 6.36 & 52.04 & 26.52 & 112815.3 & 14.20 & 46.455 \\
\hline & 100 & $116,392.3$ & 20.5 & 8.05 & 69.75 & 35.73 & 273049.5 & 20.498 & 42.630 \\
\hline
\end{tabular}

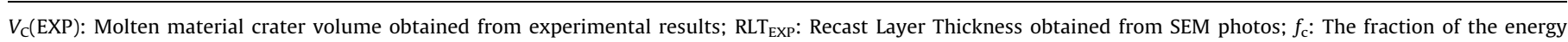

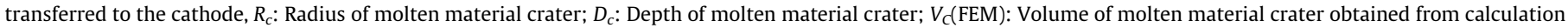

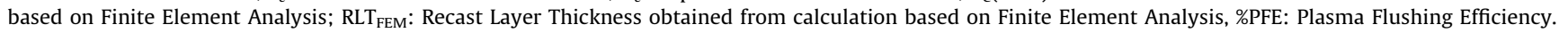


Table 4

Experimental data and theoretical features employed in regression modeling of $f_{a}$.

\begin{tabular}{|c|c|c|c|c|c|c|c|c|c|}
\hline$I(\mathrm{~A})$ & $T i(\mu \mathrm{s})$ & $V_{\mathrm{c}}(\mathrm{EXP})\left(\mu \mathrm{m}^{3}\right)$ & $\operatorname{RLT}_{\text {EXP }}(\mu \mathrm{m})$ & $f_{\mathrm{a}}$ & $R_{\mathrm{C}}(\mu \mathrm{m})$ & $D_{\mathrm{C}}(\mu \mathrm{m})$ & $V_{\mathrm{c}}(\mathrm{FEM})\left(\mu \mathrm{m}^{3}\right)$ & $\operatorname{RLT}_{\mathrm{FEM}}(\mu \mathrm{m})$ & PFE\% \\
\hline \multirow[t]{4}{*}{8} & 12.8 & 216.040 & 11.4 & 21.9 & 22.48 & 11.7 & 9287.49 & 11.428 & 2.326 \\
\hline & 25 & 184.493 & 14.4 & 28 & 29.06 & 14.54 & $19,287.48$ & 14.401 & 0.957 \\
\hline & 50 & 207.493 & 16.8 & 33.2 & 34.95 & 16.88 & $32,388.19$ & 16.772 & 0.641 \\
\hline & 100 & 190.117 & 18 & 41.7 & 44.13 & 18.08 & $55,307.77$ & 18.018 & 0.344 \\
\hline \multirow[t]{4}{*}{16} & 12.8 & 1176.415 & 14.8 & 17 & 29.43 & 15.66 & $21,305.52$ & 14.795 & 5.522 \\
\hline & 25 & 1435.332 & 20.9 & 22.1 & 39.23 & 21.5 & $51,975.05$ & 20.906 & 2.762 \\
\hline & 50 & 1090.625 & 28.2 & 29 & 53.97 & 28.44 & $130,123.1$ & 28.202 & 0.838 \\
\hline & 100 & 1037.776 & 33.7 & 36.4 & 68.84 & 33.85 & $251,976.6$ & 33.711 & 0.412 \\
\hline \multirow[t]{4}{*}{24} & 12.8 & 2418.777 & 16.8 & 14.3 & 34.04 & 18.15 & $33,035.1$ & 16.821 & 7.322 \\
\hline & 25 & 2907.071 & 24.3 & 18.9 & 45.98 & 25.15 & $83,521.02$ & 24.275 & 3.481 \\
\hline & 50 & 3643.632 & 27.3 & 21.4 & 56.6 & 27.98 & $140,799.3$ & 27.256 & 2.588 \\
\hline & 100 & 3097.773 & 40.5 & 30.7 & 80.48 & 40.83 & $415,408.3$ & 40.526 & 0.746 \\
\hline
\end{tabular}

$f_{\mathrm{a}}$ : The fraction of the energy transferred to the anode.

Table 5

Results of counting the number of pulses.

\begin{tabular}{|c|c|c|c|c|c|c|c|}
\hline$I(\mathrm{~A})$ & $T i(\mu \mathrm{s})$ & Normal-pulse (\%) & Open-circuit (\%) & Arc-discharge (\%) & Number of normal pulses & Number of open circuits & Number of arc discharges \\
\hline \multirow[t]{4}{*}{8} & 12.8 & 20 & 73 & 7 & $31,654,125$ & $11,078,944$ & $115,537,556$ \\
\hline & 25 & 24 & 62 & 14 & $20,964,057$ & $12,229,033$ & $54,157,147$ \\
\hline & 50 & 25 & 58 & 17 & $11,616,340$ & $7,899,111$ & $26,949,909$ \\
\hline & 100 & 36 & 42 & 22 & $7,253,029$ & $4,432,406$ & $8,461,867$ \\
\hline \multirow[t]{4}{*}{16} & 12.8 & 16 & 72 & 12 & $38,127,910$ & $28,595,932$ & $171,575,595$ \\
\hline & 25 & 18 & 63 & 19 & $24,821,988$ & $26,200,987$ & $86,876,958$ \\
\hline & 50 & 26 & 48 & 26 & $13,815,255$ & $13,815,255$ & $25,505,086$ \\
\hline & 100 & 34 & 43 & 23 & $7,486,255$ & $5,064,231$ & $9,467,910$ \\
\hline \multirow[t]{4}{*}{24} & 12.8 & 8 & 82 & 10 & $29,705,000$ & $37,131,250$ & $304,476,250$ \\
\hline & 25 & 13 & 68 & 19 & $23,037,995$ & $33,670,915$ & $120,506,435$ \\
\hline & 50 & 22 & 50 & 28 & $13,405,659$ & $17,061,747$ & $30,467,406$ \\
\hline & 100 & 26 & 46 & 28 & $1,662,505$ & $1,790,390$ & $2,941,355$ \\
\hline
\end{tabular}

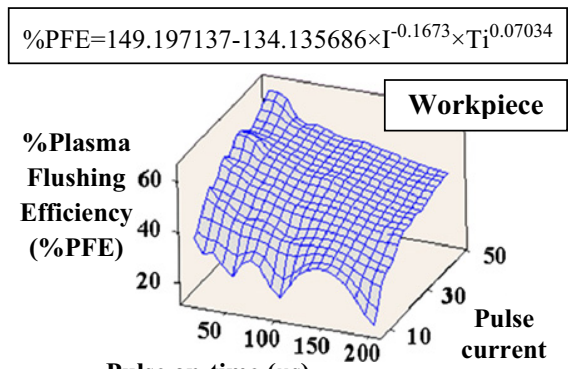

Pulse on-time ( $\mu$ s)

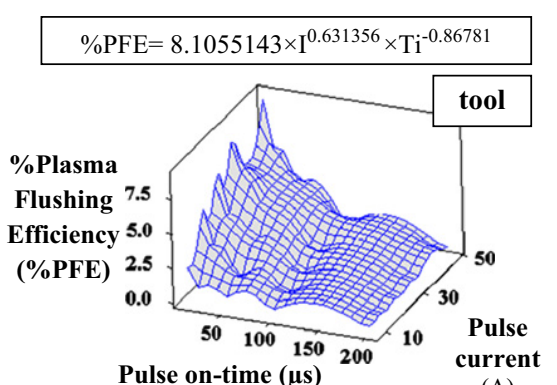

(A)

Fig. 3. Surface plot of \%PFE vs. pulse current and pulse on-time for workpiece and tool.

and current variations against time, and the FORTRAN program written to count the number of each type of pulses.

Also, the valuse of recast layer thickness (RLT) has been caculted through the following equation:

$R L T=D_{C}-\left(D_{C} \times \%\right.$ PFE $)$

Eqs. (12) and (13) represent the correlation between the $f_{\mathrm{c}}$ and $f_{\mathrm{a}}$ with the pulse current and pulse on-time achieved through the power regression modeling. What is clear is that the quota of cathode and anode from the generated heat in the plasma channel, which was previously considered to be constant for different machining setting, differ by the pulse current and pulse on time

$f_{c}=5.5998 \times I^{-0.3401} \times T i^{0.2989}$

$f_{a}=-19.2521+38.8627 \times I^{-0.2008} \times T i^{0.1889}$

Eqs. (14) and (15) represents the correlation between the \%PFE and pulse current and pulse on-time achieved through the regression modeling for AISI H13 workpiece and copper tool, respectively. Furthermore, the surface plots of \%PFE vs. pulse current and pulse on-time for both workpiece and tool are 
shown in Fig. 3.

\%PFE $($ workpiece $)=149.197137-134.135686 \times I^{-0.1673} \times T i^{0.07034}$

$\%$ PFE $($ tool $)=8.1055143 \times I^{0.631356} \times T i^{-0.86781}$

Based on the \%PFEs represented in Eqs. (14) and (15) and through utilizing Eq. (11) it becomes possible to predict the recast layer thickness for the selected pulse current and pulse on-time.

\subsection{Validation methodology}

The validation methodology in this work is based on the comparison between the empirical values of recast layer thickness ( RLT $_{\mathrm{EXP}}$ ) and the values gained by the model developed in this research ( $\left.\mathrm{RLT}_{\mathrm{FEM}}\right)$. The dependency of fraction of heat distributed into the electrodes ( $f_{\mathrm{c}}$ for cathode and $f_{\mathrm{a}}$ for anode) during each discharge to the input-parameters of process has been illustrated by Eqs. (12) and (13). To validate the accuracy of obtained results and correlations, a number of comparisons have been conducted. Having this in mind, the introduced equations in this work have been utilized to gain the values of recast layer thickness for some randomly selective setting. Also, the experiments have been done for these settings and the experimental

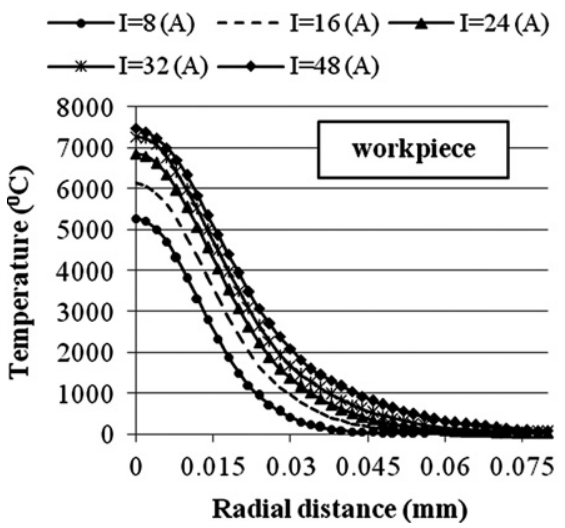

values for RLT have been achieved after EDM machining and carrying the preparation stages as described in the experimental procedure. Briefly speaking, the comparisons between the theoretical and empirical values of recast layer thickness are the method of evaluating the validity of represented model and formulas.

\section{Model validation}

So far, a model describing the thermal distribution in the electrodes during each discharge and the correlation of inputparameters of process with $f_{\mathrm{c}}$ and $f_{\mathrm{a}}$ achieved by the regression modeling through comparing the empirical and numerical values of recast layer thickness (RLT) have been represented. Fig. 4 represents the results of a typical temperature distribution simulation for $T i=12.8 \mu \mathrm{s}$. In order to evaluate the accuracy of the presented model, a number of comparisons have been conducted between the outcomes of the developed model and the experimental results. Having this in mind, based on temperature distribution profiles and utilizing the Eqs. (11)-(15) the theoretical values for a number of selective settings have been obtained. These results along with the empirical values of recast layer thickness for the same process settings are represented in Tables 6 and 7. The comparisons of theoretical and experimental

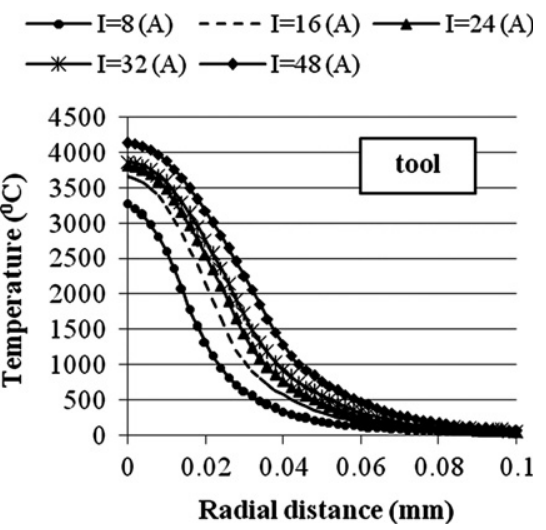

Fig. 4. Temperature distribution in radial direction of spark domain for workpiece and tool $(T i=25 \mu \mathrm{s})$.

Table 6

Comparison between the experimental and predicted values for recast layer thickness on workpiece.

\begin{tabular}{|c|c|c|c|c|c|c|c|c|c|c|}
\hline$I(\mathrm{~A})$ & $T i(\mu \mathrm{s})$ & $V_{\mathrm{c}}(\mathrm{EXP})\left(\mu \mathrm{m}^{3}\right)$ & F_Reg ${ }_{c}$ & $R_{\mathrm{C}}(\mu \mathrm{m})$ & $D_{\mathrm{C}}(\mu \mathrm{m})$ & $V_{\mathrm{c}}(\mathrm{FEM})\left(\mu \mathrm{m}^{3}\right)$ & \%PFE & $\operatorname{RLT}_{\mathrm{EXP}}(\mu \mathrm{m})$ & $\operatorname{RLT}_{\mathrm{FEM}}(\mu \mathrm{m})$ & Error (\%) \\
\hline 8 & 200 & $29,331.01$ & 13.45 & 64.88 & 34.78 & 229,970 & 14.643 & 30.1 & 29.79 & 1.03 \\
\hline 24 & 200 & $232,036.2$ & 9.259 & 90.42 & 45.29 & $581,635.9$ & 39.894 & 28.4 & 27.222 & 4.15 \\
\hline 32 & 100 & $167,093.1$ & 6.825 & 37.88 & 76.73 & $350,316.2$ & 67.48 & 19.5 & 19.812 & 1.60 \\
\hline 48 & 12.8 & $20,528.98$ & 3.2165 & 16.1 & 35.85 & $32,503.05$ & 63.16 & 6.4 & 5.931 & 7.32 \\
\hline 48 & 50 & $79,840.65$ & 4.834 & 59.38 & 27.55 & $152,588.5$ & 52.324 & 13.2 & 13.135 & 0.49 \\
\hline
\end{tabular}

F_Reg : The fraction of the energy transferred to the cathode obtained from regression modeling.

Table 7

Comparison between the experimental and predicted values for recast layer thickness on tool.

\begin{tabular}{|c|c|c|c|c|c|c|c|c|c|c|}
\hline$I(\mathrm{~A})$ & $T i(\mu \mathrm{s})$ & $V_{c}(\operatorname{EXP})\left(\mu \mathrm{m}^{3}\right)$ & F_Reg & $R_{\mathrm{C}}(\mu \mathrm{m})$ & $D_{\mathrm{C}}(\mu \mathrm{m})$ & $V_{\mathrm{c}}(\mathrm{FEM})\left(\mu \mathrm{m}^{3}\right)$ & $\% \mathrm{PFE}$ & $\operatorname{RLT}_{\text {EXP }}(\mu \mathrm{m})$ & $\operatorname{RLT}_{\mathrm{FEM}}(\mu \mathrm{m})$ & Error (\%) \\
\hline 24 & 200 & 1921.865 & 36.5953 & 104.6 & 46.82 & $804,664.2$ & 0.239 & 52 & 46.70817 & 10.18 \\
\hline 32 & 25 & 5613.386 & 16.33656 & 48.68 & 25.83 & $961,49.14$ & 5.838 & 24.1 & 24.32199 & 0.92 \\
\hline 32 & 100 & 6986.188 & 26.9907 & 88.68 & 46.08 & $569,224.7$ & 0.661 & 44.9 & 45.51445 & 1.95 \\
\hline 48 & 12.8 & 5831.84 & 9.656326 & 42.64 & 22.9 & $65,401.81$ & 8.917 & 21.7 & 20.85802 & 3.88 \\
\hline 48 & 200 & 8363.713 & 29.3375 & 136.4 & 69.88 & $2,042,215$ & 0.4095 & 71.3 & 69.59381 & 2.4 \\
\hline
\end{tabular}

F_Rega: The fraction of the energy transferred to the anode obtained from regression modeling. 
values illustrated in this table reveal that the values of RLT predicted by our model match quite closely with the experimental results for all the discharge conditions by almost a uniform margin.

\section{Results and discussion}

\subsection{Effect of input-parameters on plasma flushing efficiency}

Fig. 5 represents the effect of pulse on-time on the \%PFE for both workpiece and tool. From this figure, it became clear that plasma flushing efficiency decreases by the increase in pulse on-time.

The \%PFE is dependent on the discharge energy $(W)$, gradient of energy $(\mathrm{d} W / \mathrm{d} t)$, geometrical dimensions of gap and molten material crater, pressure of gap $(P)$, and gradient of pressure $(\mathrm{d} P / \mathrm{d} t)$. Decrease in the \%PFE by the increase in the pulse on-time could be justified in this way that the increase in the pulse ontime causes the decrease in the energy changing rate $(\mathrm{d} W / \mathrm{d} t)$ which leads to decrease in the pressure of gap $(P)$ and its gradient $(\mathrm{d} P / \mathrm{d} t)$. As a result, regarding to the mechanism of bulk boiling, the amount of molten material, which could be flushed from the molten material crater decreases and as a result, the \%PFE decreases. The decrease in the amount of molten material which sweeps away from the molten material crater by the collapse of plasma channel at the end of pulse on-time causes the increase in the recast layer thickness.

Moreover, the results show that the \%PFE of tool (anode) is much lower than that of workpiece (cathode). This phenomenon could be justified by the fact that the surface of the tool is bombarded by the electrons which could barely increase the surface temperature of tool while the surface of workpiece is bombarded by ions that not only increase the surface temperature of workpiece but causes the detachment of particles from the workpiece material making them suspended in the melting puddle. Furthermore, the present carbine in the gap through decomposition of hydrocarbon dielectric plus the free particles electronized by the free electrons in the gap move towards the anode and deposit on the surface of the molten puddle [20]. This deposited layer of carbine and electrically charged particles on the surface of molten puddle provides a protective effect through reduction of the density of copper vapor and prevents the flushing of material by the collapse of plasma channel. This layer's protective effect increases by the increase in pulse on-time as it becomes thicker [21].

On the other hand, with an increase in the pulse current and with a constant amount of pulse on-time, the gradient of energy increases, as this causes an increase in the diameter and a sharp rise in the average temperature of the plasma channel spontaneously, which leads to increase in the pressure of gap and its gradient. So, regarding about the mechanism of bulk boiling phenomenon, the amount of molten material, which is ejected from the molten puddle at the end of each discharge, increases and as a result, the \%PFE increases (Fig. 5) as the reports of Marafona and Chousal [7] prove this matter.

\subsection{Effect of input-parameters on recast layer thickness}

The increase in the thickness of recast layer by the increase in pulse on-time for both workpiece and tool can be obviously seen from the experimental results (Fig. 6). The justification for this phenomenon is that the plasma flushing efficiency has a strict effect on the recast layer thickness. With an increase in pulse on-time, plasma flushing efficiency decreases (Fig. 5), as a result,
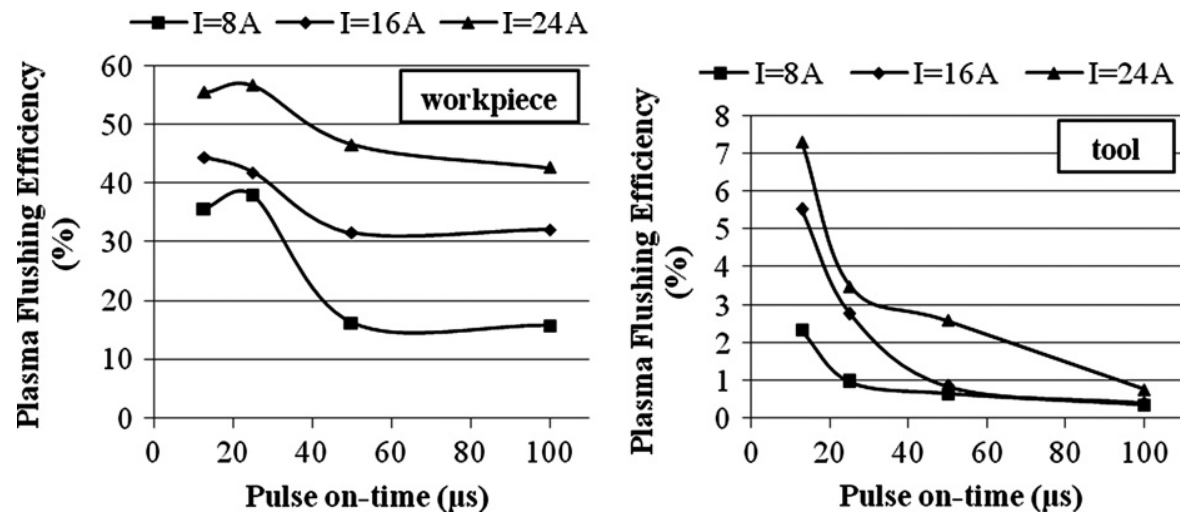

Fig. 5. Plasma flushing efficiency vs pulse on-time for workpiece and tool.
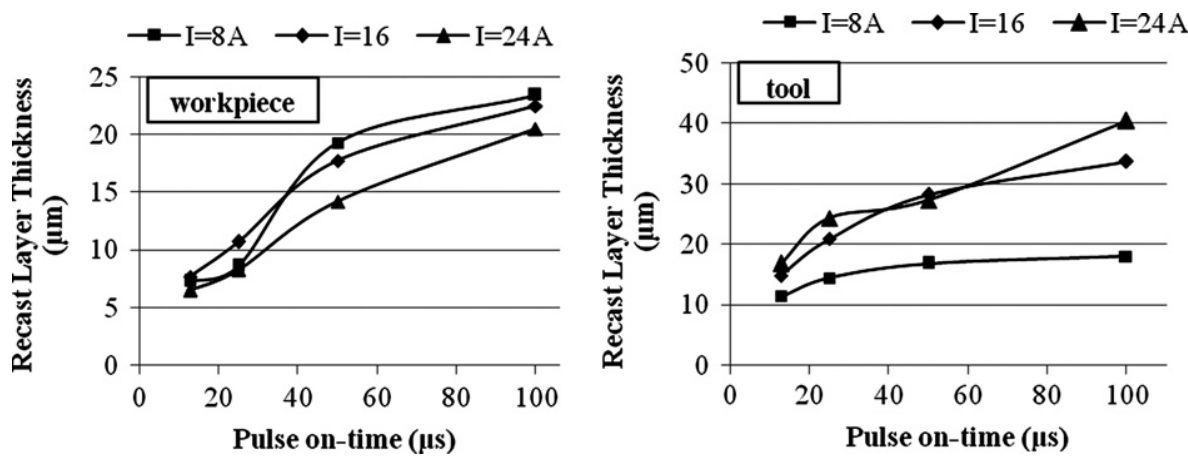

Fig. 6. Recast layer thickness vs. pulse on-time on workpiece and tool. 

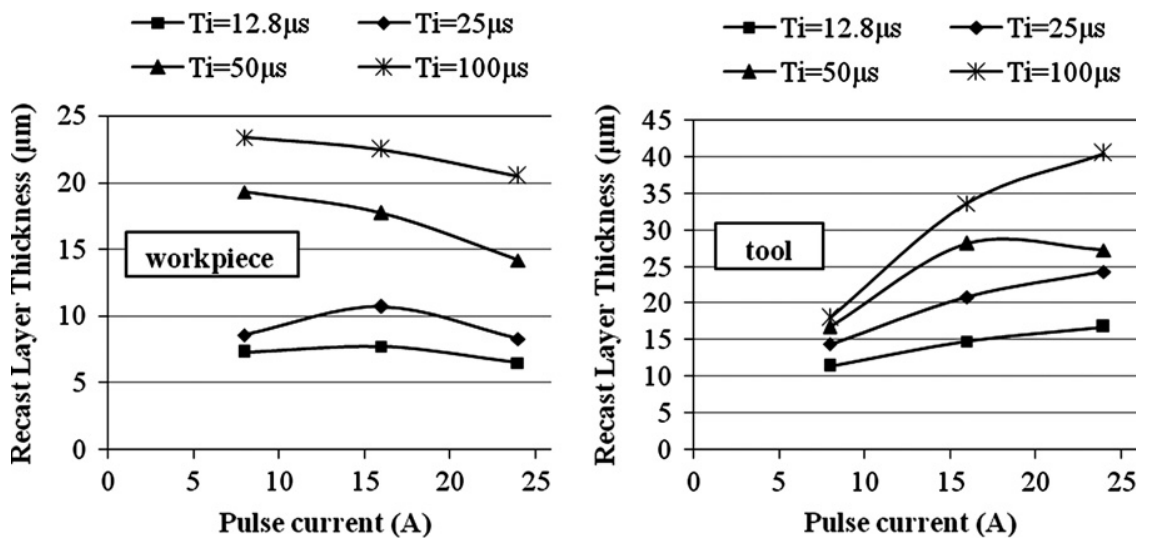

Fig. 7. Recast layer thickness vs. pulse current on workpiece and tool.

the ability of plasma channel for ejecting the molten material from the molten puddle decreases. Subsequently, this remained molten material in the molten puddle re-solidifies and forms the recast layer upon the machined surface. Furthermore, the increase of discharge duration increases the amount of the conducted heat into the workpiece during each discharge, and consequently, more underlying material is affected by the high temperature. Overly, this phenomenon causes the increase in the recast layer thickness and heat affected zone.

From Fig. 7 it is clear that, increasing the pulse current has a very small effect on the recast layer thickness of both workpiece and tool. Although an increase in pulse current leads to the increase in the dimensions of the molten crater and the heat penetrating depth, the plasma flushing efficiency increases as pulse current increases. The increase in plasma flushing efficiency causes more molten material to be swept away from the molten crater, therefore thinner layer of re-deposited material appears on the surface of workpiece. Since an increase in the penetrating depth of heat into the workpiece and plasma flushing efficiency counterbalance each other's effect, an increase in the pulse current has no significant effect on the recast layer thickness.

\section{Conclusions}

In a review of previous studies, it came into consideration that different researchers have proposed various values for the fraction of heat going into the electrodes $\left(f_{\mathrm{c}}\right.$ for cathode and $f_{\mathrm{a}}$ for anode) in their theoretical models of the process. In this work it has been demonstrated that the fraction of heat going into the electrodes is not constant but a function of input-parameters of process. The leading conclusions are as follows:

1. The results revealed the fact that the energy absorption fractions of electrodes are variable and contingent upon the process settings, as in the range of input parameters of this study the cathode fraction $\left(f_{c}\right)$ changed between 4.13 and 8.9, and the anode fraction varied from 4.13 to 36.4 .

2. Achieving accurate prediction of plasma flushing efficiency (\%PFE) is dependent upon reliable estimate of heat fraction of anode $\left(f_{\mathrm{a}}\right)$ and cathode $\left(f_{\mathrm{c}}\right)$.

3. The implication of the introduced method is able to provide reliable measures to estimate the output parameters of EDM process including the critical feature of recast layer thickness as it has been predicted with the minor prediction errors of $2.89 \%$ and $2.64 \%$ for cathode and anode, respectively.

4. The plasma flushing efficiency is influenced by the pulse current and pulse on-time, as it has a direct correlation with the prior one and a converse relationship with the latter one.

5. Recast layer thickness increases by the increase in pulse on-time for both workpiece and tool.

6. There is not much difference in the recast layer thickness for both workpiece and tool by the increase in pulse current.

\section{References}

[1] S. Abdulkareem, A.A. Khan, Z.M. Zain, Effect of machining parameters on surface roughness during wet and dry wire-EDM of stainless steel, Journal of Applied Sciences 11 (2011) 1867-1871.

[2] A.A.N. Mohammad Yeakub, K. Mustafizul, A. Erry Yulian Triblas, I. Ahmad Faris, A. Aisy Anuarin, I. Mohd Nazrol, Comparative study of conventional and micro WEDM based on machining of meso/micro sized spur gear, International Journal of Precision Engineering and Manufacturing 11 (5) (2010) 779-784.

[3] M. Boujelbene, E. Bayraktar, W. Tebni, S. Ben Salem, Influence of machining parameters on the surface integrity in electrical discharge machining, Archives of Materials Science and Engineering 37 (2009) 110-116.

[4] B. Izquierdo, J. Antonio Sánchez, N. Ortega, S. Plaza, I. Pombo, Insight into fundamental aspects of the EDM process using multi-discharge numerical simulation, International Journal of Advanced Manufacturing Technology 52 (2011) 195-206.

[5] D.D. Dibitoto, P.T. Eubank, M.R. Patel, M.A. Barrufet, Theoretical models of the electrical discharge machining process. I. A simple cathode erosion model, Journal of Applied Physics 66 (9) (1989) 4095-4103.

[6] N. Ben Salah, F. Ghanem, K. Ben Atig, Numerical study of thermal aspects of electric discharge machining process, International Journal of Machine Tools \& Manufacture 46 (2006) 908-911.

[7] J. Marafona, J.A.G. Chousal., A finite element model of EDM based on Joule effect, International Journal of Machine Tools \& Manufacture 46 (2006) 595-602.

[8] H.K. Kansal, S. Singh, P. Kumar, Numerical simulation of powder mixed electric discharge machining (PMEDM) using finite element method, Mathematical and Computer Modelling 47 (2008) 1217-1237.

[9] S.N. Joshi, S.S. Pande, Intelligent process modeling and optimization of diesinking electric discharge machining, Applied Soft Computing 11 (2011) 2743-2755.

[10] X.H. Hashimoto, M. Kunieda, N. Nishiwaki, Measurement of energy distribution in continuous EDM process, Journal of the Japan Society for Precision Engineering 62 (1996) 1141-1145.

[11] S.H. Yeo, W. Kurnia, P.C. Tan, Electro-thermal modeling of anode and cathode in micro-EDM, Journal of Physics D: Applied Physics 40 (2007) 2513-2521.

[12] Böhler Edelstahl, 〈http://www.bohler-edelstahl.at $\rangle$.

[13] Pickwick manufacturing service, 〈http://www.efunda.com/ $\rangle$.

[14] H.T. Lee, T.Y. Tai, Relationship between EDM parameters and surface crack formation, Journal of Materials Processing Technology 142 (2003) 676-683.

[15] V. Yadav, K.V. Jain, M.P. Dixit, Thermal stress due to electrical discharge machining, International Journal of Manufacturing Technology 42 (2007) $877-888$.

[16] S.N. Joshi, S.S. Pande, Thermo-physical modeling of die-sinking EDM process, Journal of Manufacturing Processes 12 (2010) 45-56.

[17] T. Ikai, K. Hashigushi, Heat input for crater formation in EDM, in: Proceedings of the International Symposium for Electro-Machining-ISEM XI, EPFL, Lausanne, Switzerland April 1995, pp. 163-170. 
[18] S. Keith Hargrove, D. Ding, Determining cutting parameters in wire EDM based on workpiece surface temperature distribution, International Journal of Advanced Manufacturing Technology 34 (2007) 296-299.

[19] K. Salonitis, A. Stournaras, P. Stavropoulos, G. Chryssolouris, Thermal modeling of the material removal rate and surface roughness for die-sinking EDM, International Journal of Advanced Manufacturing Technology 40 (2009) 316-323.
[20] Reece Roth, J., Industrial Plasma Engineering, Department of Electrical and Computer Engineering, University of Tennessee, Knoxville, Institute of Physics Publishing, 2001.

[21] M. Kunieda, T. Kobayashi, Clarifying mechanism of determining tool electrode wear ratio in EDM using spectroscopic measurement of vapor density, Journal of Materials Processing Technology 149 (2004) 284-288. 